\title{
MATRIX-VALUED BEREZIN KERNELS
}

\author{
G. VAN DIJK \\ Mathematical Institute, Leiden University \\ P.O. Box 9512, 2300 RA Leiden, The Netherlands \\ E-mail:dijk@math.leidenuniv.nl \\ M. PEVZNER \\ Université Libre de Bruxelles, CP 218 \\ Campus Plaine, 2 Bd du Triomphe, Brussels, Belgium \\ E-mail: mpevzner@ulb.ac.be
}

Introduction. Studying quantization, F. A. Berezin (see [1]) introduced a family $B_{\lambda}$ of $G$-invariant kernels on a Hermitian symmetric space $G / K$. For "large" values of the parameter $\lambda$ these kernels give rise to some positive-definite bi- $K$-invariant functions $\psi_{\lambda}$. The decomposition of $\psi_{\lambda}$ into a direct (not necessarily discrete) sum of positive-definite spherical functions can also be understood via group representation theory.

In fact, it is known (see $[3,9,14,17])$ that Berezin kernels occur in a natural way when one considers the decomposition problem for the tensor product of a holomorphic and anti-holomorphic discrete series representation of $G \times G$ restricted to $G=\operatorname{diag}(G \times G)$.

Following the same reasoning the decomposition of holomorphic discrete series representations of $G$ restricted to some "causally" symmetric subgroup $H$ (see Table 1 for the classification) is obtained using the spherical Fourier transform of the corresponding Berezin kernels (see [4]).

A logical continuation of this problem is the extension to the case of vector-valued holomorphic discrete series representations of the group $G$.

We develop a general theory for the associated matrix-valued Berezin kernels and establish some useful properties of them.

The last part of this paper is devoted to the really relevant case $G=S U(1, n), H=$ $S O_{o}(1, n)$. We consider the vector-valued holomorphic discrete series representations $\pi$ induced by the slightly modified spinor representations of the maximal compact subgroup

2000 Mathematics Subject Classification: Primary 22E30; Secondary 33C20.

Received 21 December 2000.

The paper is in final form and no version of it will be published elsewhere. 
$K$. We obtain an explicit expression, in terms of Euler Beta and Gauss Hypergeometric functions, for the decomposition spectrum of the tensor product of $\pi$ with a scalar antiholomorphic discrete series representation, considered as a representation of $G$ and the decomposition spectrum for $\pi$ when restricted to a "fully restrictive" group $H$.

We are grateful to G. Zhang for fruitful discussions and helpful remarks during the preparation of this manuscript.

M. Pevzner would like to thank the Mathematical Institute of Leiden University, where this work was done, for its hospitality.

Table 1. The irreducible causal symmetric pairs

\begin{tabular}{|c|c|c|}
\hline $\begin{array}{c}\mathfrak{g} \\
\text { compactly causal }\end{array}$ & $\begin{array}{c}\mathfrak{g}^{c} \\
\text { non-compactly causal }\end{array}$ & $\mathfrak{h}$ \\
\hline $\begin{aligned} \mathfrak{s u}(p, q) & \oplus \mathfrak{s u}(p, q) \\
\mathfrak{s o}^{*}(2 n) & \oplus \mathfrak{s o}{ }^{*}(2 n) \\
\mathfrak{s o}(2, n) & \oplus \mathfrak{s o}(2, n) \\
\mathfrak{s p}(n, \mathbf{R}) & \oplus \mathfrak{s p}(n, \mathbf{R}) \\
\mathfrak{e}_{6(-14)} & \oplus \mathfrak{e}_{6(-14)} \\
\mathfrak{e}_{7(-25)} & \oplus \mathfrak{e}_{7(-25)}\end{aligned}$ & $\begin{array}{c}\mathfrak{s l}(p+q ; \mathbf{C}) \\
\mathfrak{s o}(2 n ; \mathbf{C}) \\
\mathfrak{s o}(2+n ; \mathbf{C}) \\
\mathfrak{s p}(n, \mathbf{C}) \\
\mathfrak{e}_{6} \\
\mathfrak{e}_{7}\end{array}$ & $\begin{array}{c}\mathfrak{s u}(p, q) \\
\mathfrak{s o}^{*}(2 n) \\
\mathfrak{s o}(2, n) \\
\mathfrak{s p}(n, \mathbf{R}) \\
\mathfrak{e}_{6(-14)} \\
\mathfrak{e}_{7(-25)}\end{array}$ \\
\hline $\begin{array}{c}\mathfrak{s u}(p, q) \\
\mathfrak{s u}(n, n) \\
\mathfrak{s u}(2 p, 2 q) \\
\mathfrak{s o}^{*}(2 n) \\
\mathfrak{s o}^{*}(4 n) \\
\mathfrak{s o}(2, p+q) \\
\mathfrak{s p}(n, \mathbf{R}) \\
\mathfrak{s p}(2 n, \mathbf{R}) \\
\mathfrak{e}_{6(-14)} \\
\mathfrak{e}_{6(-14)} \\
\mathfrak{e}_{7(-25)} \\
\mathfrak{e}_{7(-25)} \\
\end{array}$ & $\begin{array}{c}\mathfrak{s l}(p+q ; \mathbf{R}) \\
\mathfrak{s u}(n, n) \\
\mathfrak{s u}^{*}(2(p+q)) \\
\mathfrak{s o}(n, n) \\
\mathfrak{s o}^{*}(4 n) \\
\mathfrak{s o}(p+1, q+1) \\
\mathfrak{s p}(n, \mathbf{R}) \\
\mathfrak{s p}(n, n) \\
\mathfrak{e}_{6(6)} \\
\mathfrak{e}_{6(-26)} \\
\mathfrak{e}_{7(-25)} \\
\mathfrak{e}_{7(7)} \\
\end{array}$ & $\begin{array}{c}\mathfrak{s o}(p, q) \\
\mathfrak{s l}(n ; \mathbf{C}) \oplus \mathbf{R} \\
\mathfrak{s p}(p, q) \\
\mathfrak{s o}(n ; \mathbf{C}) \\
\mathfrak{s u}(2 n) \oplus \mathbf{R} \\
\mathfrak{s o}(p, 1) \times \mathfrak{s o}(1, q) \\
\mathfrak{s l}(n ; \mathbf{R}) \oplus \mathbf{R} \\
\mathfrak{s p}(n, \mathbf{C}) \\
\mathfrak{s p}(2,2) \\
\mathfrak{f}_{4(-20)} \\
\mathfrak{e}_{6(-26)} \oplus \mathbf{R} \\
\mathfrak{s u}{ }^{*}(8)\end{array}$ \\
\hline
\end{tabular}

1. Structure theory. In this section we recall some structure theory, mainly following [13] and [8], Ch. VIII.

1.1. Hermitian symmetric spaces. Let $\mathfrak{g}$ be a non-compact simple real Lie algebra with complexification $\mathfrak{g}_{c}$. Let $\mathfrak{g}=\mathfrak{k} \oplus \mathfrak{p}$ be a Cartan decomposition and let $\theta$ denote the corresponding Cartan involution. Let $\mathfrak{z}$ denote the center of $\mathfrak{k}$. $\mathfrak{g}$ is said to be Hermitian if the centralizer of $\mathfrak{z}$ in $\mathfrak{g}$ is equal to $\mathfrak{k}$. The center of $\mathfrak{k}$ is one-dimensional and there is an element $Z_{0} \in \mathfrak{z}$ such that $\left(\operatorname{ad} Z_{0}\right)^{2}=-1$ on $\mathfrak{p}$. Fixing $i$ a square root of -1 , one has $\mathfrak{p}_{c}=\mathfrak{p}+i \mathfrak{p}=\mathfrak{p}_{+}+\mathfrak{p}_{-}$where $\left.\operatorname{ad} Z_{0}\right|_{\mathfrak{p}_{+}}=i,\left.\operatorname{ad} Z_{0}\right|_{\mathfrak{p}_{-}}=-i$. Then

$$
\mathfrak{g}_{c}=\mathfrak{p}_{+} \oplus \mathfrak{k}_{c} \oplus \mathfrak{p}_{-} .
$$

and $\left[\mathfrak{p}_{ \pm}, \mathfrak{p}_{ \pm}\right]=0,\left[\mathfrak{p}_{+}, \mathfrak{p}_{-}\right]=\mathfrak{k}_{c}$ and $\left[\mathfrak{k}_{c}, \mathfrak{p}_{ \pm}\right]=\mathfrak{p}_{ \pm}$. Let $G_{c}$ be a connected, simply connected Lie group with Lie algebra $\mathfrak{g}_{c}$ and $K_{c}, P_{+}, P_{-}, G, K$ the analytic subgroups correspond- 
ing to $\mathfrak{k}_{c}, \mathfrak{p}_{+}, \mathfrak{p}_{-}, \mathfrak{g}$ and $\mathfrak{k}$ respectively. Then $K_{c} P_{-}$(and $K_{c} P_{+}$) is a maximal parabolic subgroup of $G_{c}$ with split component $A=\exp i \mathbb{R} Z_{0}$. $G$ is closed in $G_{c}$.

Moreover, the exponential mapping is a diffeomorphism of $\mathfrak{p}_{-}$onto $P_{-}$and of $\mathfrak{p}_{+}$onto $P_{+}([8]$, Ch. VIII, Lemma 7.8). Furthermore:

Lemma 1.1 (see [8], Ch. VIII, Lemmæ 7.9 and 7.10). a. The mapping $(q, k, p) \mapsto q k p$ is a diffeomorphism of $P_{+} \times K_{c} \times P_{-}$onto an open dense submanifold of $G_{c}$ containing $G$. b. The set $G K_{c} P_{-}$is open in $P_{+} K_{c} P_{-}$and $G \cap K_{c} P_{-}=K$.

Thus $G / K$ is mapped on an open, bounded domain $\mathcal{D}$ in $\mathfrak{p}_{+} \cdot G$ acts on $\mathcal{D}$ via holomorphic transformations.

EXAmple. Let $\mathfrak{g}=\mathfrak{s u}(1,1)$. Then $G_{c}=S L(2, \mathbb{C})$ and $G=S U(1,1)$. Clearly $Z_{0}=$ $\frac{1}{2}\left(\begin{array}{cc}i & 0 \\ 0 & -i\end{array}\right), \mathfrak{p}_{ \pm}$are one-dimensional and generated by $\left(\begin{array}{ll}0 & 1 \\ 0 & 0\end{array}\right)$ and $\left(\begin{array}{ll}0 & 0 \\ 1 & 0\end{array}\right)$ respectively. Let $g=\left(\begin{array}{cc}\alpha & \beta \\ \bar{\beta} & \bar{\alpha}\end{array}\right) \in G$. Then the decomposition $g=q k p$ (see Lemma 1.1.a) is given by

$$
g=\left(\begin{array}{cc}
1 & \beta \bar{\alpha}^{-1} \\
0 & 1
\end{array}\right)\left(\begin{array}{cc}
\bar{\alpha}^{-1} & 0 \\
0 & \bar{\alpha}
\end{array}\right)\left(\begin{array}{cc}
1 & 0 \\
\bar{\alpha}^{-1} \bar{\beta} & 1
\end{array}\right)
$$

where $|\alpha|^{2}-|\beta|^{2}=1$. The embedding of $G / K$ into $\mathbb{C}$ is given by

$$
g \mapsto \beta \bar{\alpha}^{-1}=\zeta .
$$

Since $|\alpha|^{2}-|\beta|^{2}=1$, it follows $|\zeta|<1$. Conversely, let $|\zeta|<1$. Take then $\alpha$ such that $|\alpha|^{2}=\left(1-|\zeta|^{2}\right)^{-1}$ and let $\beta=\zeta \bar{\alpha}$. Then $\left(\begin{array}{cc}\alpha & \beta \\ \bar{\beta} & \bar{\alpha}\end{array}\right)$ is mapped onto $\zeta$. So $\mathcal{D}$ is the unit disc $"|\zeta|<1 " . G$ acts on $\mathcal{D}$ by means of fractional linear transformations

$$
g . \zeta=\frac{\alpha \zeta+\beta}{\bar{\beta} \zeta+\bar{\alpha}}, \quad g=\left(\begin{array}{cc}
\alpha & \beta \\
\bar{\beta} & \bar{\alpha}
\end{array}\right) \in G .
$$

Everywhere we shall denote $\bar{g}$ the complex conjugate of $g \in G_{c}$ with respect to $G$. So, for example, if $g=\left(\begin{array}{ll}a & 0 \\ 0 & a^{-1}\end{array}\right) \in S L(2, \mathbb{C})$, then its conjugate with respect to $S U(1,1)$ is given by $\bar{g}=\left(\begin{array}{cc}\bar{a}^{-1} & 0 \\ 0 & \bar{a}\end{array}\right)$. Notice that $\bar{P}_{+}=P_{-}$.

For $g \in P_{+} K_{c} P_{-}$we shall write $g=(g)_{+}(g)_{0}(g)_{-}$, where $(g)_{ \pm} \in P_{ \pm},(g)_{0} \in K_{c}$. For $g \in G_{c}, z \in \mathfrak{p}_{+}$such that $g$. $\exp z \in P_{+} K_{c} P_{-}$we define

$$
\begin{aligned}
\exp g(z) & =(g \cdot \exp z)_{+}, \\
J(g, z) & =(g \cdot \exp z)_{0} .
\end{aligned}
$$

$J(g, z) \in K_{c}$ is called the canonical automorphic factor of $G_{c}$ (terminology of Satake).

Lemma 1.2 (see [13], Ch. II, Lemma 5.1). J satisfies

(i) $J(g, o)=(g)_{0}$, for $g \in P_{+} K_{c} P_{-}$,

(ii) $J(k, z)=k$ for $k \in K_{c}, z \in \mathfrak{p}_{+}$.

If for $g_{1}, g_{2} \in G_{c}$ and $z \in \mathfrak{p}_{+}, g_{1}\left(g_{2}(z)\right)$ and $g_{2}(z)$ are defined, then $\left(g_{1} g_{2}\right)(z)$ is also defined and

(iii) $J\left(g_{1} g_{2}, z\right)=J\left(g_{1}, g_{2}(z)\right) J\left(g_{2}, z\right)$. 
For $z, w \in \mathfrak{p}_{+}$satisfying $(\exp \bar{w})^{-1} \cdot \exp z \in P_{+} K_{c} P_{-}$we define

$$
\begin{aligned}
K(z, w) & =J\left((\exp \bar{w})^{-1}, z\right)^{-1} \\
& =\left((\exp \bar{w})^{-1} \cdot \exp z\right)_{0}^{-1} .
\end{aligned}
$$

This expression is always defined for $z, w \in \mathcal{D}$, for then

$$
(\exp \bar{w})^{-1} \cdot \exp z \in{\overline{\left(G K_{c} P_{-}\right)}}^{-1} G K_{c} P_{-}=P_{+} K_{c} G K_{c} P_{-}=P_{+} K_{c} P_{-} .
$$

$K(z, w)$, defined on $\mathcal{D} \times \mathcal{D}$, is called the canonical kernel on $\mathcal{D}$ ( by Satake). $K(z, w)$ is holomorphic in $z$, anti-holomorphic in $w$, with values in $K_{c}$. Here are a few properties:

Lemma 1.3 (see [13], Ch. II, Lemma 5.2). (i) $K(z, w)=\overline{K(w, z)}^{-1}$ if $K(z, w)$ is defined,

(ii) $K(o, w)=K(z, o)=1$ for $z, w \in \mathfrak{p}_{+}$.

If $g(z), \bar{g}(w)$ and $K(z, w)$ are defined, then $K(g(z), \bar{g}(w))$ is also defined and one has:

(iii) $K(g(z), \bar{g}(w))=J(g, z) K(z, w) \overline{J(\bar{g}, w)}^{-1}$,

Lemma 1.4 (see [13], Ch. II, Lemma 5.3). For $g \in G_{c}$ the Jacobian of the holomorphic mapping $z \mapsto g(z)$, when it is defined, is given by

$$
\operatorname{Jac}(z \mapsto g(z))=A d_{\mathfrak{p}_{+}}(J(g, z)) .
$$

For any holomorphic character $\chi: K_{c} \mapsto \mathbb{C}$ we define:

$$
\begin{aligned}
j_{\chi}(g, z) & =\chi(J(g, z)), \\
k_{\chi}(z, w) & =\chi(K(z, w)) .
\end{aligned}
$$

Since $\chi(\bar{k})=\overline{\chi(k)}^{-1}$ we have:

$$
\begin{aligned}
k_{\chi}(z, w) & =\overline{k_{\chi}(w, z)}, \\
k_{\chi}(g(z), \bar{g}(w)) & =j_{\chi}(g, z) k_{\chi}(z, w) \overline{j_{\chi}(\bar{g}, w)}
\end{aligned}
$$

in place of Lemma (1.3) (i) and (iii).

The character $\chi_{1}(k)=\operatorname{det} \operatorname{Ad}_{\mathfrak{p}_{+}}(k),\left(k \in K_{c}\right)$ is of particular importance. We call the corresponding $j_{\chi_{1}}, k_{\chi_{1}}: j_{1}$ and $k_{1}$. Notice that

$$
j_{1}(g, z)=\operatorname{det}(\operatorname{Jac}(z \mapsto g(z))) .
$$

EXAMPLE. $\mathfrak{g}=\mathfrak{s u}(1,1)$. For $g=\left(\begin{array}{cc}\alpha & \beta \\ \bar{\beta} & \bar{\alpha}\end{array}\right)$ in $S U(1,1)$ one has

$$
J(g, z)=\left(\begin{array}{cc}
(\bar{\beta} z+\bar{\alpha})^{-1} & 0 \\
0 & (\bar{\beta} z+\bar{\alpha})
\end{array}\right), K(z, w)=\left(\begin{array}{cc}
(1-z \bar{w}) & 0 \\
0 & (1-z \bar{w})^{-1}
\end{array}\right)
$$

and $\chi_{1}\left(\begin{array}{cc}\alpha & 0 \\ 0 & \alpha^{-1}\end{array}\right)=\alpha^{2},\left(\alpha \in \mathbb{C}^{*}\right)$, so

$$
j_{1}(g, z)=(\bar{\beta} z+\bar{\alpha})^{-2}, \quad k_{1}(z, w)=(1-\bar{z} w)^{2} .
$$

Because of $(10),\left|k_{1}(z, z)\right|^{-1} d \mu(z)$, where $d \mu(z)$ is the Euclidean measure on $\mathfrak{p}_{+}$, is a $G$-invariant measure on $\mathcal{D}$. Indeed: 


$$
\begin{aligned}
d \mu(g(z)) & =\left|j_{1}(g, z)\right|^{2} d \mu(z), \\
k_{1}(g(z), g(z)) & =j_{1}(g, z) k_{1}(z, z) \overline{j_{1}(g, z)}, \quad \text { for } g \in G .
\end{aligned}
$$

(see (1.9)). One can actually show that $k_{1}(z, z)>0$ on $\mathcal{D}$ ([13], Ch. II, Lemma 5.8).

For the list of groups $G$ considered here, we refer to the upper part of Table 1, right column.

1.2. Symmetric spaces of Hermitian type. Let $\mathfrak{g}, \mathfrak{g}_{c}, G, G_{c}, \ldots$ be as in section 1.1 . We add to $\mathfrak{g}$ an involutive automorphism $\sigma$, commuting with the Cartan involution $\theta$. Let $\mathfrak{g}=\mathfrak{h} \oplus \mathfrak{q}$ be the decomposition of $\mathfrak{g}$ into +1 and -1 eigenspaces of $\sigma$.

The Lie algebra $\mathfrak{g}$ is said to be of Hermitian type if $\mathfrak{g}$ is Hermitian and, in addition, $Z_{0} \in \mathfrak{q} \cap \mathfrak{k}$. There are several other terminologies in use; the most closely related to us is: $\mathfrak{g}$ is a compactly causal Lie algebra.

The involution $\sigma$ is extended to $\mathfrak{g}_{c}$ and $G_{c}$ and leaves $G$ invariant. Let $H$ denote the closed subgroup of $G$ consisting of the fixed points of $\sigma$. The Lie algebra of $H$ is $\mathfrak{h}$.

Now observe that, since $\sigma\left(Z_{0}\right)=-Z_{0}, \sigma\left(\mathfrak{p}_{+}\right)=\mathfrak{p}_{-}$. Since $\overline{\mathfrak{p}}_{+}=\mathfrak{p}_{-}$, we see that $\bar{\sigma}$, defined by $\bar{\sigma}(X)=\sigma(\bar{X})$, leaves $\mathfrak{p}_{+}$and $\mathfrak{p}_{-}$invariant. Set

$$
\mathfrak{p}_{ \pm}^{\bar{\sigma}}=\left\{X \in \mathfrak{p}_{ \pm}: \bar{\sigma}(X)=X\right\}
$$

Then clearly $\operatorname{dim}_{\mathbb{R}} \mathfrak{p}_{+}^{\bar{\sigma}}=\operatorname{dim}_{\mathbb{R}} \mathfrak{p}_{-}^{\bar{\sigma}}=\operatorname{dim}_{\mathbb{C}} \mathfrak{p}_{+}$, since $\bar{\sigma}$ is a conjugation.

It is clear that $\bar{\sigma}(\mathcal{D})=\mathcal{D}$. Set $\mathcal{D}^{\bar{\sigma}}$ for the set of fixed points of $\bar{\sigma}$ in $\mathcal{D}$. Since $\bar{\sigma}(H)=H$ it easily follows that $H / H \cap K$ can be identified with an open submanifold of $\mathcal{D}^{\bar{\sigma}}$. The proof is according to the same lines as in Lemma (1.1). The real "ball" $\mathcal{D}^{\bar{\sigma}}$ is an interesting object; one can actually show that $H$ acts transitively on it.

EXAMPLE. $\mathfrak{g}=\mathfrak{s u}(1,1), \sigma(X)=\bar{X}, \mathfrak{h}=\mathfrak{s o}(1,1) . H=S O(1,1), h \in H$ is of the form $\left(\begin{array}{c}\cosh t \sinh t \\ \sinh t \cosh t\end{array}\right), t \in \mathbb{R}$.

Now $\mathcal{D}=\{z \in \mathbb{C}:|z|<1\}$, so $\mathcal{D}^{\bar{\sigma}}=(-1,1) \subset \mathbb{R}$. This is clearly the same as $H . o=\{\tanh t: t \in \mathbb{R}\}$.

It is clear that $\left|k_{1}(z, z)\right|^{-1 / 2} d \nu(z)$, where $d \nu(z)$ is a Euclidean measure on $\mathcal{D}^{\bar{\sigma}}$, is a $H$-invariant measure on $\mathcal{D}^{\bar{\sigma}}$. The proof is along the same line as in section 1.1.

For the spaces we are talking about, see Table 1 (lower part). This table also includes a list of non-compactly causal Lie algebras $\mathfrak{g}^{c}=\mathfrak{h} \oplus i \mathfrak{q}$ which has been discussed in [5]. Table 1 is taken from [7].

\section{Bergman kernel of a holomorphic discrete series representation}

2.1. The matrix-valued holomorphic discrete series. Let $\tau$ be an irreducible holomorphic representation of $K_{c}$ on a finite dimensional complex vector space $V$ with scalar product $\langle\mid\rangle$, such that $\tau_{\left.\right|_{K}}$ is unitary.

LEMMA 2.1. $\tau^{*}(k)=\tau(\bar{k})^{-1}$ for $k \in K_{c}$.

This follows easily by writing $k=k_{o} \cdot \exp i X$ with $k_{O} \in K, X \in \mathfrak{k}$ and using that $\tau_{\left.\right|_{K}}$ is unitary.

Call $\pi_{\tau}=\operatorname{Ind}_{K}^{G} \tau$ and set $V_{\tau}$ for the space of representation of $\pi_{\tau}$. Then $V_{\tau}$ consists of maps $f: G \mapsto V$ satisfying 
(i) $f$ measurable,

(ii) $f(g k)=\tau^{-1}(k) f(g)$ for $g \in G, k \in K$,

(iii) $\int_{G / K}\|f(g)\|^{2} d \dot{g}<\infty$,

where $\|f(g)\|^{2}=\langle f(g) \mid f(g)\rangle$ and $d \dot{g}$ an invariant measure on $G / K$. Let us identify $G / K$ with $\mathcal{D}$ and $d \dot{g}$ with $d_{*} z=k_{1}(z, z)^{-1} d \mu(z)$. Then $V_{\tau}$ can be identified with a space of maps on $\mathcal{D}$, setting

$$
\varphi(z)=\tau(J(g, o)) f(g),
$$

if $z=g(o), f \in V_{\tau}$. Indeed, the right-hand side of (11) is clearly right $K$-invariant. The inner product becomes

$$
(\varphi \mid \psi)=\int_{\mathcal{D}}\left\langle\tau^{-1}(J(g, o)) \varphi(z) \mid \tau^{-1}(J(g, o)) \psi(z)\right\rangle d_{*} z .
$$

Since $\tau^{-1}(J(g, o))^{*} \tau^{-1}(J(g, o))=\tau^{-1}\left(J(g, o) \overline{J(g, o)}^{-1}\right)=\tau^{-1}(K(z, z))$ by Lemma $(1.3)$, we may also write

$$
(\varphi \mid \psi)=\int_{\mathcal{D}}\left\langle\tau^{-1}(K(z, z)) \varphi(z) \mid \psi(z)\right\rangle d_{*} z
$$

The $G$-action on the new space is given by

$$
\pi_{\tau}(g) \varphi(z)=\tau^{-1}\left(J\left(g^{-1}, z\right)\right) \varphi\left(g^{-1}(z)\right) \quad(g \in G, z \in \mathcal{D}) .
$$

Now we restrict to the closed subspace of holomorphic maps and call the resulting Hilbert space $\mathcal{H}_{\tau}$. The space $\mathcal{H}_{\tau}$ is $\pi_{\tau}(G)$-invariant. We assume that $\mathcal{H}_{\tau} \neq\{0\}$; see however section 2.3 .

The pair $\left(\pi_{\tau}, \mathcal{H}_{\tau}\right)$ is called a holomorphic discrete series of $G$.

In a similar way we can define the anti-holomorphic discrete series. We therefore start with $\bar{\tau}$ instead of $\tau$ and take anti-holomorphic maps. Then

$$
\pi_{\bar{\tau}}(g) \psi(z)=\bar{\tau}^{-1}\left(J\left(g^{-1}, z\right)\right) \psi\left(g^{-1}(z)\right) .
$$

for $\psi \in \mathcal{H}_{\bar{\tau}}$. One easily sees that $\mathcal{H}_{\bar{\tau}}=\overline{\mathcal{H}}_{\tau}$ and $\pi_{\bar{\tau}}=\bar{\pi}_{\tau}$ in the usual sense.

2.2. The Bergman kernel. The Hilbert space $\mathcal{H}_{\tau}$ (see section 2.1) is known to have a reproducing (or Bergman) kernel $\mathcal{K}_{\tau}(z, w)$. Its definition is as follows. Set

$$
E_{z}: \varphi \mapsto \varphi(z) \quad\left(\varphi \in \mathcal{H}_{\tau}\right)
$$

for $z \in \mathcal{D}$. Then $E_{z}: \mathcal{H}_{\tau} \mapsto V$ is a continuous linear operator, and $\mathcal{K}_{\tau}(z, w)=E_{z} E_{w}^{*}$, being a $\operatorname{End}(V)$-valued kernel, holomorphic in $z$, anti-holomorphic in $w$. In more detail:

$$
\langle\varphi(w) \mid \xi\rangle=\int_{\mathcal{D}}\left\langle\tau^{-1}(K(z, z)) \varphi(z) \mid \mathcal{K}_{\tau}(z, w) \xi\right\rangle d_{*} z
$$

for any $\varphi \in \mathcal{H}_{\tau}, \xi \in V$ and $w \in \mathcal{D}$.

Since $\mathcal{H}_{\tau}$ is a $G$-module, one easily gets the following transformation property for $\mathcal{K}_{\tau}(z, w)$ :

$$
\mathcal{K}_{\tau}(g(z), g(w))=\tau(J(g, z)) \mathcal{K}_{\tau}(z, w) \tau(\overline{J(g, w)})^{-1} \quad(g \in G, z, w \in \mathcal{D}) .
$$

Now consider $H(z, w)=\mathcal{K}_{\tau}(z, w) \cdot \tau^{-1}(K(z, w))$.

Clearly $H(g(z), g(w))=\tau(J(g, z)) H(z, w) \tau^{-1}(J(g, z))$ for all $z, w \in \mathcal{D}$. So, setting $z=w=o, g \in K$ we see that $H(o, o)$ is a scalar operator, and hence $H(z, z)=H(o, o)$ 
is. But then $H(z, w)=H(o, o)$. So, we get

$$
\mathcal{K}_{\tau}(z, w)=c \cdot \tau(K(z, w)),
$$

where $c$ is a scalar. The way of obtaining (17) is similar to [13] Ch II, Lemma 6.1.

The same reasoning yields that $\pi_{\tau}$ is irreducible. Indeed, if $\mathcal{H} \subset \mathcal{H}_{\tau}$ is a closed invariant subspace, then $\mathcal{H}$ has a reproducing kernel, say $K_{\mathcal{H}}$ and it follows that $K_{\mathcal{H}}=c \mathcal{K}_{\tau}$, so either $\mathcal{H}=\{0\}$ or $\mathcal{H}=\mathcal{H}_{\tau}$.

2.3. Examples. In this section we consider several representations $\tau$ of $K$ (or $K_{c}$ ) with $\mathcal{H}_{\tau} \neq\{0\}$ and more precisely the spinor representations. We were inspired by the paper [12] of Pedon.

The group $\operatorname{Ad}(K)$ acts irreducibly on $\mathfrak{p}$, but its action is reducible on $\mathfrak{p}_{c}$, while splitting into two irreducible subspaces $\mathfrak{p}_{c}=\mathfrak{p}_{+} \oplus \mathfrak{p}_{-}$. An inner product on $\mathfrak{p}_{c}$ is given by $\langle X \mid Y\rangle=$ $B(X, \bar{Y})$, where $B$ is the Killing form of $\mathfrak{g}_{c}$. It is clear that $\mathfrak{p}_{+}$and $\mathfrak{p}_{-}$are orthogonal with respect to this inner product. Moreover, the map $X \rightarrow \bar{X}$, which is anti-linear, of $\mathfrak{p}_{+}$into $\mathfrak{p}_{-}$, gives an isomorphism of $\operatorname{Ad}(k)_{\left.\right|_{\mathfrak{p}_{-}}}$and $\overline{\operatorname{Ad}(\bar{k})_{\left.\right|_{\mathfrak{p}_{+}}}}\left(k \in K_{c}\right)$. So we have

$$
\overline{\operatorname{Ad}(k)_{\left.\right|_{\mathfrak{p}_{-}}} \bar{X}}=\operatorname{Ad}(\bar{k})_{\left.\right|_{\mathfrak{p}_{+}}} X \quad\left(X \in \mathfrak{p}_{+}\right) .
$$

The latter is equal to $\operatorname{Ad}\left(k^{-1}\right)_{\left.\right|_{\mathfrak{p}_{+}} ^{*}}^{*} X$.

Let $n=\operatorname{dim} \mathfrak{p}_{-}$. Define for $k \in K_{c}$ the holomorphic representations

$$
\text { (i) } \quad \tau_{n}(k)=\operatorname{det}_{\mathbb{C}} \operatorname{Ad}(k)_{\left.\right|_{\mathfrak{p}_{-}}} \quad \text { (scalar valued), }
$$$$
\text { (ii) } \quad \tau_{1}(k)=\operatorname{Ad}(k)_{\left.\right|_{\mathfrak{p}_{-}}} \text {on } \mathfrak{p}_{-},
$$

$$
\text { (iii) } \quad \tau_{r}(k)=\bigwedge^{r} \operatorname{Ad}(k)_{\left.\right|_{\mathfrak{p}_{-}}} \text {on } \bigwedge^{r} \mathfrak{p}_{-}, \quad(1 \leq r \leq n) .
$$

The representations $\tau_{n}$ and $\tau_{1}$ are irreducible, while $\tau_{r}$ certainly is in case $G=S U(1, n)$ (see Section 5.2). Let us assume that $\tau_{r}$ is irreducible, $1 \leq r \leq n$.

Next, set for $\ell \in \mathbb{Z}$ and $k \in K_{c}$,

$$
\begin{aligned}
(i) & \tau_{n, \ell}(k)=\tau_{n}(k)^{\ell}, \\
(\text { ii }) & \tau_{1, \ell}(k)=\tau_{n}(k)^{\ell-1} \tau_{1}(k), \\
(\text { iii }) & \tau_{r, \ell}(k)=\tau_{n}(k)^{\ell-1} \tau_{r}(k)
\end{aligned}
$$

Then $\tau_{n, \ell}$ gives rise to so-called scalar holomorphic discrete series of $G$ on $\mathcal{H}_{n, \ell}$. Clearly

$$
\tau_{n, l}^{-1}(K(z, z))=j_{1}(K(z, z))^{-\ell}=k_{1}(z, z)^{\ell} .
$$

So

$$
\mathcal{H}_{n, \ell} \neq\{0\} \quad \text { for } \ell \geq 1 \text {. }
$$

In a similar way, applying that the eigenvalues of $\operatorname{Ad} K(z, z)_{\left.\right|_{\mathfrak{p}_{+}}}$are real, positive and bounded by 1 (see [13], Ch. II, Lemma 5.8), we get:

$$
\mathcal{H}_{r, \ell} \neq\{0\} \quad \text { for } \ell \geq 2 \text {. }
$$

For $r=n$, see (25). 
3. Study of the tensor product of a matrix-valued holomorphic and a scalar anti-holomorphic discrete series representation. Let $\tau$ be one of the representations (19)-(21) and $\tau_{\ell}=\tau_{n}^{\ell-1} \tau$ one of the representations (22)-(24).

Set $\pi_{\ell}^{\tau}=\pi_{\tau_{\ell}}$ and $\mathcal{H}_{\ell}^{\tau}=\mathcal{H}_{\tau_{\ell}}$. If $\tau=\tau_{n}$ then we simply write $\pi_{\ell}$ and $\mathcal{H}_{\ell}$. We will study the tensor product

$$
\pi_{\ell+1}^{\tau} \widehat{\otimes}_{2} \bar{\pi}_{\ell}
$$

as a representation of $G$ and finally determine its expansion into irreducible unitary representations. Let us assume $\ell \geq 1$.

3.1. The restriction map. For $f \in \mathcal{H}_{\ell+1}^{\tau}$ and $g \in \mathcal{H}_{\ell}$ define the map

$$
A_{\ell}^{\tau}: f(z) \otimes \bar{g}(w) \rightarrow f(z) \bar{g}(z) k_{1}(z, z)^{\ell}
$$

of $\mathcal{H}_{\ell+1}^{\tau} \widehat{\otimes}_{2} \overline{\mathcal{H}}_{\ell}$ into the space of $V$-valued distributions on $\mathcal{D}$, denoted by $D^{\prime}(\mathcal{D}, V)$. Writing general elements in $\mathcal{H}_{\ell+1}^{\tau} \widehat{\otimes}_{2} \overline{\mathcal{H}}_{\ell}$ as $F(z, w)$ we have

$$
A_{\ell}^{\tau} F(z)=F(z, z) k_{1}(z, z)^{\ell} .
$$

Here $A_{\ell}^{\tau} F(z)$ is seen as the distribution

$$
\left\langle A_{\ell}^{\tau} F \mid \varphi\right\rangle=\int_{\mathcal{D}}\left\langle\tau^{-1}(K(z, z)) F(z) \mid \varphi(z)\right\rangle d_{*} z \quad(\varphi \in D(\mathcal{D}, V)) .
$$

Notice that $A_{\ell}^{\tau}$ is an intertwining operator:

$$
A_{\ell}^{\tau} \circ\left(\pi_{\ell+1}^{\tau}(g) \otimes \pi_{\ell}(g)\right)=\pi_{\tau}(g) \underset{3}{\operatorname{i} r c} A_{\ell}^{\tau} \quad(g \in G) .
$$

We are going to compute

$$
\left(A_{\ell}^{\tau}\right)^{*}: D(\mathcal{D}, V) \rightarrow \mathcal{H}_{\ell+1}^{\tau} \widehat{\otimes}_{2} \overline{\mathcal{H}}_{\ell} .
$$

Let $h \in D(\mathcal{D}, V)$ be a $V$-valued test function on $\mathcal{D}$. Then $\left(A_{\ell}^{\tau}\right)^{*} h(z, w)$ is an element of the right-hand side of (31), holomorphic in $z$, anti-holomorphic in $w$.

Set $\mathcal{K}_{\tau}(z, w)=\tau(K(z, w))$ and let $K_{\ell}(z, w)$ be the reproducing kernel of $\mathcal{H}_{\ell}$. Then

$$
K_{\ell+1}^{\tau}(z, w)=c_{\ell}^{\tau} \mathcal{K}_{\tau}(z, w) K_{\ell}(z, w)
$$

is the reproducing kernel of $\mathcal{H}_{\ell}^{\tau}$ where $c_{\ell}^{\tau}$ is a constant, depending on $\tau$ and $\ell$. Observe that $K_{\ell}(z, w)=c_{\ell}^{1} k_{1}(z, w)^{-\ell}$.

We have for $F \in \mathcal{H}_{\ell+1}^{\tau} \widehat{\otimes}_{2} \overline{\mathcal{H}_{\ell}}$ and $h \in D(\mathcal{D}, V)$ :

$$
\begin{aligned}
& \left\langle\left(A_{\ell}^{\tau}\right)^{*} h \mid F\right\rangle=\left(h, A_{\ell}^{\tau} F\right)=\int_{\mathcal{D}}\left\langle\tau^{-1}(K(z, z)) h(z) \mid F(z, z)\right\rangle k_{1}(z, z)^{\ell} d_{*} z \\
= & \int_{\mathcal{D}} \int_{\mathcal{D}}\left\langle\tau^{-1}(K(z, z)) h(z) \mid F(z, w)\right\rangle K_{\ell}(z, w) k_{1}(w, w)^{\ell} k_{1}(z, z)^{\ell} d_{*} w d_{*} z .
\end{aligned}
$$

We have to write (33) in the form

$$
\int_{\mathcal{D}} \int_{\mathcal{D}}\left\langle\tau^{-1}(K(z, z))\left(A_{\ell}^{\tau}\right)^{*} h(z, w) \mid F(z, w)\right\rangle k_{1}(w, w)^{\ell} k_{1}(z, z)^{\ell} d_{*} w d_{*} z .
$$

Therefore we apply the reproducing kernel property (15) for $F(\cdot, w)$, so (33) becomes:

$$
\begin{aligned}
& \int_{\mathcal{D}} \int_{\mathcal{D}} \int_{\mathcal{D}}\left\langle K_{\ell+1}^{\tau}\left(w^{\prime}, z\right) \tau^{-1}(K(z, z)) h(z) \mid \tau^{-1}\left(K\left(w^{\prime}, w^{\prime}\right)\right) F\left(w^{\prime}, w\right)\right\rangle \\
\cdot & K_{\ell}(z, w) k_{1}(w, w)^{\ell} k_{1}(z, z)^{\ell} k_{1}\left(w^{\prime}, w^{\prime}\right)^{\ell} d_{*} w^{\prime} d_{*} w d_{*} z .
\end{aligned}
$$


So:

$$
\left(A_{\ell}^{\tau}\right)^{*} h(z, w)=\int_{\mathcal{D}} K_{\ell+1}^{\tau}\left(z, z^{\prime}\right) \tau^{-1}\left(K\left(z^{\prime}, z^{\prime}\right)\right) h\left(z^{\prime}\right) k_{1}\left(z^{\prime}, z^{\prime}\right)^{\ell} K_{\ell}\left(z^{\prime}, w\right) d_{*} z^{\prime} .
$$

3.2. The Berezin kernel. Hence we obtain for $A_{\ell}^{\tau}\left(A_{\ell}^{\tau}\right)^{*}$ the following expression. Let $f \in D(\mathcal{D}, V)$, then:

$$
\begin{aligned}
& A_{\ell}^{\tau}\left(A_{\ell}^{\tau}\right)^{*} f(z)=k_{1}(z, z)^{\ell} \int_{\mathcal{D}} K_{\ell+1}^{\tau}\left(z, z^{\prime}\right) \tau^{-1}\left(K\left(z^{\prime}, z^{\prime}\right)\right) f\left(z^{\prime}\right) k_{1}\left(z^{\prime}, z^{\prime}\right)^{\ell} K_{\ell}\left(z^{\prime}, w\right) d_{*} z^{\prime} \\
= & c_{\ell}^{\prime} c_{\ell}^{\tau} \int_{\mathcal{D}} \mathcal{K}_{\tau}\left(z, z^{\prime}\right) \tau^{-1}\left(K\left(z^{\prime}, z^{\prime}\right)\right) k_{1}\left(z, z^{\prime}\right)^{-\ell} k_{1}\left(z^{\prime}, z\right)^{-\ell} k_{1}(z, z)^{\ell} k_{1}\left(z^{\prime}, z^{\prime}\right)^{\ell} f\left(z^{\prime}\right) d_{*} z^{\prime} .
\end{aligned}
$$

We see that $A_{\ell}^{\tau}\left(A_{\ell}^{\tau}\right)^{*}$ is a kernel operator on $D(\mathcal{D}, V)$ with the kernel

$$
\begin{aligned}
B_{\ell}^{\tau}\left(z, z^{\prime}\right)= & c_{\ell}^{\prime} c_{\ell}^{\tau} \tau^{-1}(K(z, z)) \mathcal{K}_{\tau}\left(z, z^{\prime}\right) \tau^{-1}\left(K\left(z^{\prime}, z^{\prime}\right)\right) \\
& \cdot k_{1}\left(z, z^{\prime}\right)^{-\ell} k_{1}\left(z^{\prime}, z\right)^{-\ell} k_{1}(z, z)^{\ell} k_{1}\left(z^{\prime}, z^{\prime}\right)^{\ell} .
\end{aligned}
$$

Observe that $B_{\ell}^{\tau}$ is an Hermitian kernel.

$B_{\ell}^{\tau}$ has the following transformation property under $G$ :

$$
B_{\ell}^{\tau}\left(g(z), g\left(z^{\prime}\right)\right)=\tau^{-1}(J(g, z))^{*} B_{\ell}^{\tau}\left(z, z^{\prime}\right) \tau^{-1}\left(J\left(g, z^{\prime}\right)\right) .
$$

So, consider

$$
F_{\ell}^{\tau}\left(g, g^{\prime}\right)=\tau(J(g, o))^{*} B_{\ell}^{\tau}\left(g(o), g^{\prime}(o)\right) \tau\left(J\left(g^{\prime}, o\right)\right) .
$$

This map is $G$-invariant. Let

$$
\psi_{\ell}^{\tau}(g)=F_{\ell}^{\tau}(e, g) \quad(g \in G) .
$$

So, $\psi_{\ell}^{\tau}(g)=B_{\ell}^{\tau}(o, g(o)) \tau(J(g, o))$; and it satisfies

$$
\psi_{\ell}^{\tau}\left(k g k^{\prime}\right)=\tau(k) \psi_{\ell}^{\tau}(g) \tau\left(k^{\prime}\right) \quad\left(g \in G, k, k^{\prime} \in K\right) .
$$

Furthermore:

$$
B_{\ell}^{\tau}(o, g(o))=c_{\ell}^{\prime} c_{\ell}^{\tau} k_{1}(z, z)^{\ell} \tau^{-1}(K(z, z))
$$

if $z=g(o)$. So,

$$
\psi_{\ell}^{\tau}(g)=c_{\ell}^{\prime} c_{\ell}^{\tau} k_{1}(g(o), g(o))^{\ell} \tau^{*^{-1}}(J(g, o))
$$

Define for $\lambda \in \mathbb{R}$,

$$
B_{\lambda}^{\tau}\left(z, z^{\prime}\right)=\tau^{-1}(K(z, z)) \mathcal{K}_{\tau}\left(z, z^{\prime}\right) \tau^{-1}\left(K\left(z^{\prime}, z^{\prime}\right)\right) \cdot\left\{\frac{k_{1}(z, z) k_{1}\left(z^{\prime}, z^{\prime}\right)}{k_{1}\left(z^{\prime}, z\right) k_{1}\left(z, z^{\prime}\right)}\right\}^{\lambda} .
$$

$B_{\lambda}^{\tau}$ is a matrix-valued Berezin kernel. It has the same properties as in (36). In a similar way we can define the function $\psi_{\lambda}^{\tau}$ associated with the Berezin kernel by

$$
\psi_{\lambda}^{\tau}(g)=k_{1}(g(o), g(o))^{\lambda} \tau^{*}\left(J(g, o)^{-1}\right) \quad(g 1 n G) .
$$

Remarks. 1. For any $\lambda \geq 1$ we can define, in an obvious way, the generalized Fock spaces $\mathcal{H}_{\lambda+1}^{\tau}$. These spaces have reproducing kernels $K_{\lambda+1}^{\tau}$ and the above theory leads in a similar way to the definition of $B_{\lambda}^{\tau}$.

2. $\psi_{\lambda}^{\tau}$ is a positive-definite function since $A_{\lambda}^{\tau}\left(A_{\lambda}^{\tau}\right)^{*}$ is positive-definite for $\lambda \geq 1$.

3. $\psi_{\lambda}^{\tau} \in L^{1} \cap L^{2}(G, V)$ for $\lambda \geq 1$.

4. $A_{\lambda}^{\tau}$ is a bounded linear operator from $\mathcal{H}_{\lambda+1}^{\tau} \widehat{\otimes}_{2} \overline{\mathcal{H}_{\lambda}}$ into $V_{\tau}$; moreover $A_{\lambda}^{\tau}$ is one-to-one for $\lambda \geq 1$. The proofs are similar to the case $\tau \equiv 1$ (see [4]). 
3.3. Restriction to real bounded domains. For $f \in \mathcal{H}_{\ell+1}^{\tau}$ define the map

$$
\mathcal{A}_{\ell}^{\tau}: f(z) \rightarrow f(x) k_{1}(x, x)^{\ell / 2}
$$

of $\mathcal{H}_{\ell+1}^{\tau}$ into $D^{\prime}\left(\mathcal{D}^{\bar{\sigma}}, V\right)$ (so $x \in \mathcal{D}^{\bar{\sigma}}$ ). The map $\mathcal{A}_{\ell}^{\tau}$ is clearly one-to-one and continuous. Moreover $\mathcal{A}_{\ell}^{\tau}$ is an intertwining operator, at least for $\ell \in m \mathbb{N}$ where $m$ is a positive integer satisfying $\tau_{n}^{\ell}(k)^{m}=1$ for $k \in K \cap H$ :

$$
\mathcal{A}_{\ell}^{\tau} \circ \pi_{\ell+1}^{\tau}(h)=\pi_{\tau}(h) \circ \mathcal{A}_{\ell}^{\tau} \quad(h \in H) .
$$

The existence of $m$ follows from the fact that the center of $K$ has finite intersection with $K \cap H$ because $G / H$ is a compactly causal space. Strictly speaking $\pi_{\tau}(h)$ has not been defined; here is the definition:

$$
\pi_{\tau}(h) \varphi(x)=\tau^{-1}(J(h, x)) \varphi(h . x) \quad\left(h \in H, x \in \mathcal{D}^{\bar{\sigma}}, \varphi \in D^{\prime}(\mathcal{D}, V)\right) .
$$

As in Section 3 we can determine $\left(\mathcal{A}_{\ell}^{\tau}\right)^{*}$ and then $\mathcal{A}_{\ell}^{\tau}\left(\mathcal{A}_{\ell}^{\tau}\right)^{*}$, which again is a kernel operator on $D\left(\mathcal{D}^{\bar{\sigma}}, V\right)$ with kernel proportional to:

$$
\mathcal{B}_{\ell}^{\tau}\left(x, x^{\prime}\right)=\tau^{-1}(K(x, x)) \mathcal{K}_{\tau}\left(x, x^{\prime}\right) \tau^{-1}\left(K\left(x^{\prime}, x^{\prime}\right)\right) \cdot\left\{\frac{k_{1}(x, x) k_{1}\left(x^{\prime}, x^{\prime}\right)}{k_{1}\left(x^{\prime}, x\right) k_{1}\left(x, x^{\prime}\right)}\right\}^{\ell / 2}
$$

Remark that $\mathcal{B}_{\ell}^{\tau}\left(x, x^{\prime}\right)=B_{\ell / 2}^{\tau}\left(x, x^{\prime}\right)$ for $x, x^{\prime} \in \mathcal{D}^{\bar{\sigma}}$.

$\mathcal{B}_{\ell}^{\tau}$ has the following transformation property under $H$ :

$$
\mathcal{B}_{\ell}^{\tau}\left(h(x), h\left(x^{\prime}\right)\right)=\tau^{-1}(J(h, x))^{*} \mathcal{B}_{\ell}^{\tau}\left(x, x^{\prime}\right) \tau^{-1}\left(J\left(h, x^{\prime}\right)\right) .
$$

We can associate with $\mathcal{B}_{\ell}^{\tau}$ a positive-definite matrix-valued function

$$
\Psi_{\ell}^{\tau}(h)=k_{1}(h(o), h(o))^{\ell / 2} \tau^{*^{-1}}(J(h, o))=\psi_{\ell / 2}^{\tau}(h) \quad(h \in H) .
$$

In a similar way as in Section 3 we can define for $\lambda \in \mathbb{R}$

$$
\mathcal{B}_{\lambda}^{\tau}\left(x, x^{\prime}\right)=B_{\lambda / 2}^{\tau}\left(x, x^{\prime}\right) \text { and } \Psi_{\lambda}^{\tau}(h)=\psi_{\lambda / 2}^{\tau}(h)
$$

for $x, x^{\prime} \in \mathcal{D}^{\bar{\sigma}}$ and $h \in H$. The function $\Psi_{\lambda}^{\tau}$ satisfies

$$
\Psi_{\lambda}^{\tau}\left(k h k^{\prime}\right)=\tau(k) \Psi_{\lambda}^{\tau}(h) \tau\left(k^{\prime}\right) \quad\left(h \in H, k, k^{\prime} \in K \cap H\right) .
$$

4. The cases $S U(1, n)$ and $S O_{o}(1, n)$. Here we shall determine $\psi_{\lambda}^{\tau}$ for $S U(1, n)$ and, by restriction, for $S O_{o}(1, n)$, and compute its spherical Fourier transforms.

4.1. Structure theory. We begin with the recollection of some structure theory of the groups in the title.

Let $\mathbb{F}$ denote one of the fields $\mathbb{R}$ or $\mathbb{C}$ and define the sesquilinear form

$$
[x, y]=\bar{y}_{o} x_{o}-\bar{y}_{1} x_{1}-\ldots-\bar{y}_{n} x_{n}
$$

on $\mathbb{F}^{n+1}$. Let $G=S U(1, n, \mathbb{F})$ be the group of $(n+1) \times(n+1)$ matrices with coefficients in $\mathbb{F}$ and determinant 1 , which preserve this form. In case of $\mathbb{F}=\mathbb{R}$ we take the connected component of $G$.

The Lie algebra of $G$ consists of the matrices $X$ of the form

$$
X=\left(\begin{array}{cc}
Z_{1} & Z_{2} \\
{ }^{t} \bar{Z}_{2} & Z_{3}
\end{array}\right)
$$


with $Z_{1}(1 \times 1), Z_{2}(1 \times n), Z_{3}(n \times n)$ matrices, satisfying: $Z_{1}$ and $Z_{2}$ anti-Hermitian, $\operatorname{tr}\left(Z_{1}+Z_{3}\right)=0, Z_{2}$ arbitrary. Let $J$ be the $(n+1) \times(n+1)$ matrix: $J=\operatorname{diag}(-1,1, \ldots, 1)$ and set

$$
\vartheta X=J X J .
$$

Then $\vartheta$ is a Cartan involution with Cartan decomposition $\mathfrak{g}=\mathfrak{k} \oplus \mathfrak{p}$. Here $\mathfrak{k}$ is the Lie algebra of $K=S(U(1) \times U(n))$ (respectively $K=S O(n))$.

Clearly

$$
\begin{aligned}
& \mathfrak{k}=\left\{\left(\begin{array}{cc}
Z_{1} & 0 \\
0 & Z_{3}
\end{array}\right): Z_{1}, Z_{3} \text { anti-Hermitian; } \operatorname{tr}\left(Z_{1}+Z_{3}\right)=0\right\}, \\
& \mathfrak{p}=\left\{\left(\begin{array}{cc}
0 & Z_{2} \\
{ }^{t} \bar{Z}_{2} & 0
\end{array}\right): Z_{2} \text { arbitrary } 1 \times n \text { matrix }\right\} .
\end{aligned}
$$

Let $L$ be the following element of $\mathfrak{g}$ :

$$
L=\left(\begin{array}{lll}
0 & 0 & 1 \\
0 & O & 0 \\
1 & 0 & 0
\end{array}\right)
$$

We have $L \in \mathfrak{p}$ and $\mathfrak{a}=\mathbb{R} L$ is a maximal Abelian subspace of $\mathfrak{p}$. We are going to diagonalize the operator ad $L$. The centralizer of $L$ in $\mathfrak{k}$ is

$$
\mathfrak{m}=\left\{\left(\begin{array}{lll}
u & 0 & 0 \\
0 & v & 0 \\
0 & 0 & u
\end{array}\right): u \in \mathbb{F}, u+\bar{u}=0, v \in U(n-1, \mathbb{F}), 2 u+\operatorname{tr} v=0\right\} .
$$

Let $\alpha=1$. The nonzero eigenvalues of ad $L$ are $\pm \alpha$ if $\mathbb{F}=\mathbb{R}$ and $\pm \alpha, \pm 2 \alpha$ if $\mathbb{F}=\mathbb{C}$. The space $\mathfrak{g}_{\alpha}$ consists of the matrices

$$
X=\left(\begin{array}{ccc}
0 & z^{*} & 0 \\
z & O & -z \\
0 & z^{*} & 0
\end{array}\right)
$$

where $z$ is a matrix of type $(n-1,1)$ with coefficients in $\mathbb{F}$ and with $z^{*}=-{ }^{t} \bar{z}$. The dimension of $\mathfrak{g}_{\alpha}$ is equal to $m_{\alpha}=d(n-1)$ (where $d=1$ if $\mathbb{F}=\mathbb{R}, d=2$ if $\mathbb{F}=\mathbb{C}$ ). The space $\mathfrak{g}_{2 \alpha}$ consists of the matrices of the form

$$
X=\left(\begin{array}{ccc}
w & 0 & -w \\
0 & O & 0 \\
w & 0 & -w
\end{array}\right)
$$

with $w \in \mathbb{F}, w+\bar{w}=0$. The dimension of $\mathfrak{g}_{2 \alpha}$ is equal to $m_{2 \alpha}=d-1$. We have $\mathfrak{g}=\mathfrak{g}_{-2 \alpha}+\mathfrak{g}_{-\alpha}+\mathfrak{a}+\mathfrak{m}+\mathfrak{g}_{\alpha}+\mathfrak{g}_{2 \alpha}$. Let $A$ be the subgroup exp $\mathfrak{a}$. This is the subgroup of the matrices

$$
a_{t}=\left(\begin{array}{ccc}
\cosh t & 0 & \sinh t \\
0 & I & 0 \\
\sinh t & 0 & \cosh t
\end{array}\right)
$$

where $t$ is a real number. The centralizer of $A$ in $K$ is the subgroup $M$ of the matrices

$$
\left(\begin{array}{lll}
u & 0 & 0 \\
0 & v & 0 \\
0 & 0 & u
\end{array}\right)
$$


with $u \in \mathbb{F},|u|=1(u=1$ if $\mathbb{F}=\mathbb{R}), v \in U(n-1, \mathbb{F}), u^{2} \operatorname{det} v=1$. The Lie algebra of $M$ is $\mathfrak{m}$.

The subspace $\mathfrak{n}=\mathfrak{g}_{\alpha}+\mathfrak{g}_{2 \alpha}$ is a nilpotent subalgebra. Set $N=\exp \mathfrak{n}$. This is the subgroup of the matrices

$$
n(w, z)=\left(\begin{array}{ccc}
1+w-\frac{1}{2}[z, z] & z^{*} & -w+\frac{1}{2}[z, z] \\
z & I & -z \\
w-\frac{1}{2}[z, z] & z^{*} & 1-w+\frac{1}{2}[z, z]
\end{array}\right)
$$

with $w \in \mathbb{F}, w+\bar{w}=0$ and with $z$ a matrix of type $(n-1,1)$ with coefficients in $\mathbb{F}$, $z^{*}=-{ }^{t} \bar{z}$, and if

$$
z=\left(\begin{array}{c}
z_{2} \\
\vdots \\
z_{n}
\end{array}\right), \quad z^{\prime}=\left(\begin{array}{c}
z_{2}^{\prime} \\
\vdots \\
z_{n}^{\prime}
\end{array}\right),
$$

then $\left[z, z^{\prime}\right]=-\bar{z}_{2}^{\prime} z_{2}-\ldots-\bar{z}_{n}^{\prime} z_{n}$. The composition law in $N$ is the following:

$$
n(w, z) \cdot n\left(w^{\prime}, z^{\prime}\right)=n\left(w+w^{\prime}+\Im\left[z, z^{\prime}\right], z+z^{\prime}\right) .
$$

The subgroup $A$ normalizes $N$ :

$$
a_{t} n(w, z) a_{-t}=n\left(e^{2 t} w, e^{t} z\right) .
$$

Let $2 \rho$ be the trace of the restriction of ad $L$ to $\mathfrak{n}$ :

$$
\rho=\frac{1}{2}\left(m_{\alpha}+m_{2 \alpha}\right)=\frac{1}{2} d(n+1)-1 .
$$

We have the Iwasawa decomposition $G=K A N=N A K$. Each $g \in G$ can uniquely be written as $g=k a_{t(g)} n$ accordingly. One has the corresponding integral formula

$$
\int_{G} f(g) d g=\int_{K A N} f\left(k a_{t} n\right) e^{2 \rho t} d k d t d n
$$

for $f \in D(G)$. This is also equal to

$$
\int_{N A K} f\left(n a_{t} k\right) e^{-2 \rho t} d n d t d k .
$$

Here $d n=d z d w(n=n(w, z))$ and $d k$ is the normalized Haar measure on $K$. Observe that $N A$ parameterizes $\mathcal{D} \simeq G / K$.

Moreover, we have the Cartan decomposition $G=K A_{+} K$ where

$$
A_{+}=\left\{a_{t}: t \geq 0\right\}
$$

and, after $d g$ is normalized according to (52), the corresponding integral formula is

$$
\int_{G} f(g) d g=\int_{K} \int_{0}^{\infty} \int_{K} f\left(k a_{t} k^{\prime}\right) \delta(t) d k d t d k^{\prime} .
$$

Here $\delta(t)=2 \frac{\pi^{n}}{\Gamma(n)}(\sinh t)^{2(n-1)} \sinh 2 t$.

Let $\mathbb{F}=\mathbb{C}$. Then $\mathfrak{g}_{c}=\mathfrak{s l}(n+1, \mathbb{C})$ and

$$
\mathfrak{p}_{c}=\left\{\left(\begin{array}{cc}
0 & { }^{t} X \\
Y & 0
\end{array}\right): X, Y \text { arbitrary } n \times 1 \text { matrices over } \mathbb{C}\right\} .
$$


If we take $Z_{O}=\operatorname{diag}\left(-i \frac{n}{n+1}, \frac{i}{n+1}, \ldots, \frac{i}{n+1}\right)$ in the center of $\mathfrak{k}$, then $\mathfrak{p}_{+}=\left\{\left(\begin{array}{cc}0 & 0 \\ Y & 0\end{array}\right)\right.$ : $\left.Y \in \mathbb{C}^{n}\right\}$. Obviously $K_{c}=S(G L(1, \mathbb{C}) \times G L(n, \mathbb{C}))$ and $\mathfrak{p}_{-}=\left\{\left(\begin{array}{ll}0 & X \\ 0 & 0\end{array}\right):{ }^{t} X \in \mathbb{C}^{n}\right\}$. Now let $\left(\begin{array}{ll}a & b \\ c & d\end{array}\right) \in G$, with $a(1 \times 1), b(1 \times n), c(n \times 1)$ and $d(n \times n)$ matrices. Then we have, following Lemma (1.1):

$$
\left(\begin{array}{ll}
a & b \\
c & d
\end{array}\right)=\left(\begin{array}{cc}
1 & 0 \\
c / a & 0
\end{array}\right)\left(\begin{array}{cc}
a & 0 \\
0 & d-a^{-1} c \cdot b
\end{array}\right)\left(\begin{array}{cc}
1 & b / a \\
0 & 1
\end{array}\right) .
$$

Furthermore, $\mathcal{D} \simeq\left\{z \in \mathbb{C}:\left|z_{1}\right|^{2}+\ldots+\left|z_{n}\right|^{2}<1\right\}$ and because

$$
\left(\begin{array}{ll}
a & b \\
c & d
\end{array}\right)\left(\begin{array}{ll}
1 & 0 \\
z & 0
\end{array}\right)=\left(\begin{array}{cc}
a+b \cdot z & b \\
c+d z & d
\end{array}\right)
$$

the action of $G$ on $\mathcal{D}$ is given by

$$
g . z=(c+d z)(a+b \cdot z)^{-1} .
$$

Moreover, if $g=\left(\begin{array}{ll}a & b \\ c & d\end{array}\right)$, then

$$
J(g, o)=\left(\begin{array}{cc}
a & 0 \\
0 & d-a^{-1} c \cdot b
\end{array}\right)
$$

and

$$
k_{1}(z, z)=\left(1-\|z\|^{2}\right)^{(n+1)}=|a|^{-2(n+1)} \text { if } z=\text { g.o. }
$$

4.2. The choice of $\tau$. Clearly $K_{c}$ acts on $\mathfrak{p}_{-}$(see Section 4.1) by $\operatorname{Ad}(k) X=a^{t} d^{-1} X$ if $k=\left(\begin{array}{ll}a & 0 \\ 0 & d\end{array}\right)$. Let us denote the associated representation of $K_{c}$ on $\bigwedge^{r} \mathfrak{p}_{-} \simeq \bigwedge^{r} \mathbb{C}^{n}$ by $\tau_{r}, 1 \leq r \leq n$.

According to Pedon: $\tau_{r}$ is irreducible (Proposition 2.1 of [12]). Observe that $\operatorname{Ad}(m) e_{n}$ $=e_{n}$ for $m \in M$, moreover $\mathfrak{p}_{1}=\operatorname{span}\left\{e_{1}, \ldots, e_{n-1}\right\}$ is $\operatorname{Ad}(M)$-invariant, $M$ acting by $\operatorname{Ad}(m) X_{1}=u \bar{v} X_{1}\left(X_{1} \in \mathfrak{p}_{1}\right)$ if $m=\left(\begin{array}{lll}u & 0 & 0 \\ 0 & v & 0 \\ 0 & 0 & u\end{array}\right) \in M$.

Call $\sigma_{p}$ the associated irreducible representation of $M$ on $\bigwedge^{p} \mathfrak{p}_{1}, 1 \leq p \leq n-1$. Let $\sigma_{0}=i d$. Then, according to [12], Proposition 3.1, we have:

LEMMA 4.1.

$$
\begin{aligned}
\left.\tau_{r}\right|_{M} & =\sigma_{r} \oplus \sigma_{r-1} \quad(1 \leq r \leq n-1), \\
\left.\tau_{n}\right|_{M} & =\sigma_{n-1} .
\end{aligned}
$$

Let us now consider the restriction of $\tau_{r}$ to $S O(n) \simeq\{1\} \times S O(n) \subset S O(1, n)$ and maintain the same notation for this representation. Unfortunately, $\tau_{r}$ does not always remain an irreducible representation of $S O(n)$. The following is conveniently recollected in [12], Proposition 3.2.

LEMMA 4.2. (i) $\left.\tau_{r}\right|_{S O(n)}$ is irreducible if $r \neq \frac{n}{2}$.

(ii) If $n$ is even, then $\left.\tau_{\frac{n}{2}}\right|_{S O(n)} \simeq \tau_{\frac{n}{2}}^{+} \oplus \tau_{\frac{n}{2}}^{-}$, the two factors being irreducible and inequivalent; they correspond to the decomposition $\bigwedge^{\frac{n}{2}} \mathbb{C}^{n}=\bigwedge^{\frac{n}{2}} \mathbb{C}_{+}^{n} \oplus \bigwedge^{\frac{n}{2}} \mathbb{C}_{-}^{n}$ into eigenspaces 
for the Hodge operator *. Precisely

$$
*= \pm i^{\left(\frac{n}{2}\right)^{2}} \operatorname{Id}=\left\{\begin{array}{ll} 
\pm \mathrm{Id} & \text { for } \frac{n}{2} \text { even } \\
\pm i \mathrm{Id} & \text { for } \frac{n}{2} \text { odd }
\end{array} \text { on } \bigwedge^{\frac{n}{2}} \mathbb{C}_{ \pm}^{n}\right.
$$

(iii) The Hodge operator $*$ induces an equivalence $\left.\left.\tau_{r}\right|_{S O(n)} \sim \tau_{n-r}\right|_{S O(n)}$. We can therefore restrict to $0 \leq r \leq \frac{n}{2}$.

About the $M_{o}$-decomposition we have $\left(M_{o}=\left\{\left(\begin{array}{lll}1 & 0 & 0 \\ 0 & v & 0 \\ 0 & 0 & 1\end{array}\right): v \in S O(n-1)\right\}\right)$, according to Pedon:

LEMMA 4.3. Let $1 \leq r \leq \frac{n}{2}$.

(i) If $r \neq \frac{n-1}{2}, \frac{n}{2}$ then $\bigwedge^{r} \mathbb{C}^{n}=\left(\bigwedge^{r-1} \mathbb{C}^{n}\right) \wedge e_{n} \oplus \bigwedge^{r} \mathbb{C}^{n-1}$, so

$$
\left.\tau_{r}\right|_{M_{o}}=\left.\left.\sigma_{r-1}\right|_{M_{o}} \oplus \sigma_{r}\right|_{M_{o}} .
$$

The factors occurring in the decomposition are irreducible and inequivalent.

(ii) If $r=\frac{n-1}{2}$ then $\left.\tau_{r}\right|_{M_{o}}=\sigma_{r-\left.1\right|_{M_{o}}} \oplus \sigma_{r}^{+} \oplus \sigma_{r}^{-}$is a decomposition into irreducible inequivalent factors $\left(\sigma_{\left.r\right|_{M_{o}}}\right.$ is the decomposition into eigenspaces for the Hodge operator).

(iii) If $r=\frac{n}{2}$ then $\left.\tau_{r}\right|_{M_{o}}=\left.\left.\tau_{r}^{+}\right|_{M_{o}} \tilde{\oplus} \tau_{r}^{-}\right|_{M_{o}}=\left.\left.\sigma_{r-1}\right|_{M_{o}} \tilde{\oplus} \sigma_{r}\right|_{M_{o}}$ are two decompositions into equivalent irreducible factors.

4.3. Spherical functions of type $\tau$. Let $\tau$ be an arbitrary irreducible unitary representation of $K$ on the vector space $V_{\tau}$. It is a general fact that $\tau_{\left.\right|_{M}}$ splits multiplicity free (cf. [10]). Let $\sigma$ occur in $\tau_{\left.\right|_{M}}$. Then

$$
P_{\sigma}=d_{\sigma} \int_{M} \tau\left(m^{-1}\right) \bar{\chi}_{\sigma}(m) d m
$$

where $d_{\sigma}=\operatorname{degree}(\sigma), \chi_{\sigma}$ is the character of $\sigma$ and $d m$ the normalized Haar measure of $M$, is the projection of $V_{\tau}$ onto the subspace of vectors which transform under $M$ like $\sigma$.

There is a general formula for spherical functions of type $\tau$, based on the Iwasawa decomposition $G=N A K, g=n(g) a_{t(g)} \kappa(g)(g \in G)$.

For any irreducible representation $\sigma$ contained in $\tau_{\left.\right|_{M}}$ we define the spherical function of type $\tau$ by

$$
\Phi_{\mu, \sigma}^{\tau}(x)=\frac{d_{\tau}}{d_{\sigma}} \int_{K} \tau\left(\kappa\left(k x^{-1}\right)\right)^{-1} P_{\sigma} \tau(k) e^{-\mu t\left(k x^{-1}\right)} d k,
$$

where $x \in G, \mu \in \mathbb{C}, d_{\tau}=\operatorname{degree}(\tau)$. See Warner [16] (here $G=K A N$ is used).

We refer to Pedon's work for explicit formulæ [12]. Also Plancherel formulæ are given for $\tau=\tau_{r}$ in this paper.

Let $f \in L^{1}\left(G, \operatorname{End}\left(V_{\tau}\right)\right)$ satisfy $f\left(k x k^{\prime}\right)=\tau(k) f(x) \tau\left(k^{\prime}\right)$ for all $x \in G$ and $k, k^{\prime} \in K$. Later on we shall take $f=\psi_{\lambda}^{\tau}$ for $\lambda \geq 1$.

The Fourier transform of $f$ is given by

$$
\widehat{f}(\sigma, \mu)=\int_{G} f(x) \Phi_{\mu, \sigma}^{\tau}\left(x^{-1}\right) d x .
$$

Since $\widehat{f}(\sigma, \mu)$ commutes with $\tau(k)(k \in K)$, it is a scalar operator, so $\widehat{f}(\sigma, \mu)=\frac{1}{d_{\tau}} \operatorname{tr} \widehat{f}(\sigma, \mu)$. 
We get

$$
\begin{aligned}
\widehat{f}(\sigma, \mu) & =\frac{1}{d_{\tau}} \operatorname{tr} \int_{K} \int_{G} f(x) \tau(\kappa(k x))^{-1} P_{\sigma} \tau(k) e^{-\mu t(k x)} d x d k \\
& =\frac{1}{d_{\tau}} \operatorname{tr} \int_{K} \int_{G} \tau\left(k^{-1}\right) f(x) \tau(\kappa(x))^{-1} P_{\sigma} \tau(k) e^{-\mu t(x)} d x d k \\
& =\frac{1}{d_{\tau}} \operatorname{tr} \int_{G / K} f(x) \tau(\kappa(x))^{-1} e^{-\mu t(x)} d x \cdot P_{\sigma} .
\end{aligned}
$$

Finally, using $G=N A K$ and the fact that $a^{-2 \rho} d n d a d k=d a d n d k$, we have

$$
\widehat{f}(\sigma, \mu)=\frac{1}{d_{\tau}} \operatorname{tr} \int_{N} \int_{-\infty}^{\infty} f\left(n a_{t}\right) e^{-(\mu+2 \rho) t} d t d n \cdot P_{\sigma} .
$$

Observe that $\widehat{f}(\sigma, \mu)$ commutes with $\tau(m), m \in M$, so $\widehat{f}(\sigma, \mu)$ is a scalar, depending on $\sigma$ and $\mu$. Hence

$$
\widehat{f}(\sigma, \mu)=\int_{N} \int_{-\infty}^{\infty} f\left(n a_{t}\right) e^{-(\mu+2 \rho) t} d t d n \cdot P_{\sigma} .
$$

4.4. The Fourier transform of $\psi_{\lambda}^{\tau}$. We begin by determining the spherical Fourier transform of $\psi_{\lambda}^{\tau}$ on $G=S U(1, n)$, for $\tau=\tau_{1}$, the representation of $K_{c}=S(G L(1, \mathbb{C}) \times$ $G L(n, \mathbb{C}))$ on $\mathbb{C}^{n}$ given by $\tau\left(\begin{array}{ll}a & 0 \\ 0 & d\end{array}\right)=a^{t} d^{-1}$. Then $\tau^{*}\left(\begin{array}{ll}a & 0 \\ 0 & d\end{array}\right)^{-1}=\bar{a}^{-1} \bar{d}$. Hence

$$
\psi_{\lambda}^{\tau}\left(\begin{array}{ll}
a & b \\
c & d
\end{array}\right)=|a|^{-2(n+1) \lambda} \bar{a}^{-1}\left(\bar{d}-\bar{a}^{-1} \bar{c} \cdot \bar{b}\right) .
$$

To compute the Fourier transform of $\psi_{\lambda}^{\tau}$ we apply (61). The representation $\tau_{\left.\right|_{M}}$ splits into $\sigma_{o}\left(\right.$ on $\left.\mathbb{C} e_{n}\right)$ and $\sigma_{1}\left(\right.$ on $\left.\operatorname{span}\left(e_{1}, \ldots, e_{n-1}\right)\right)$. We write down the expression for $\psi_{\lambda}^{\tau}\left(n a_{t}\right)$. We have:

$$
\begin{aligned}
\psi_{\lambda}^{\tau}\left(n a_{t}\right)= & \left|\cosh t-e^{-t}\left(w+\frac{1}{2}[z, z]\right)\right|^{-2(n+1) \lambda} \cdot\left[\cosh t-e^{-t}\left(w+\frac{1}{2}[z, z]\right)\right]^{-1} \\
& \cdot\left\{\left(\begin{array}{cc}
I & -\bar{z} e^{-t} \\
\bar{z}^{*} & \cosh t+e^{-t}\left(w+\frac{1}{2}[z, z]\right)
\end{array}\right)-\left[\cosh t-e^{-t}\left(w+\frac{1}{2}[z, z]\right)\right]^{-1}\right. \\
& \left.\cdot\left(\begin{array}{c}
\sinh t-e^{-t}\left(w+\frac{1}{2}[z, z]\right)
\end{array}\right) \cdot\left(\bar{z}^{*}, \sinh t+e^{-t}\left(w+\frac{1}{2}[z, z]\right)\right)\right\}
\end{aligned}
$$

Since $\widehat{\psi}_{\lambda}^{\tau}(\sigma, \mu)$ is a scalar operator, it is sufficient to compute the action on one vector. If $\sigma=\sigma_{o}$ we take $e_{n}$, if $\sigma=\sigma_{1}$ we take $e_{1}$. We have:

$$
\begin{aligned}
& \left\langle\psi_{\lambda}^{\tau}\left(n a_{t}\right) e_{n} \mid e_{n}\right\rangle= \\
& \left|\cosh t-e^{-t}\left(w+\frac{1}{2}[z, z]\right)\right|^{-2(n+1) \lambda}\left[\cosh t-e^{-t}\left(w+\frac{1}{2}[z, z]\right)\right]^{-1} \\
& \cdot\left\{\left[\cosh t+e^{-t}\left(w+\frac{1}{2}[z, z]\right)\right]-\left[\cosh t-e^{-t}\left(w+\frac{1}{2}[z, z]\right)\right]^{-1}\right. \\
& \left.\cdot\left(\sinh t-e^{-t}\left(w+\frac{1}{2}[z, z]\right)\right)\left(\sinh t+e^{-t}\left(w+\frac{1}{2}[z, z]\right)\right)\right\} . \\
& \left\langle\psi_{\lambda}^{\tau}\left(n a_{t}\right) e_{1} \mid e_{1}\right\rangle=
\end{aligned}
$$




$$
\begin{aligned}
& \left|\cosh t-e^{-t}\left(w+\frac{1}{2}[z, z]\right)\right|^{-2(n+1) \lambda}\left[\cosh t-e^{-t}\left(w+\frac{1}{2}[z, z]\right)\right]^{-1} \\
& \cdot\left\{1+\left[\cosh t-e^{-t}\left(w+\frac{1}{2}[z, z]\right)\right]^{-1} \cdot\left|z_{2}\right|^{2} e^{-t}\right\} .
\end{aligned}
$$

Now we do the same for $\tau=\tau_{r}, 1<r<n$. Then $\left.\tau\right|_{M}$ splits into $\sigma_{r-1}$ and $\sigma_{r}$. We get for $\sigma_{r-1}$ :

$$
\begin{aligned}
& \left\langle\psi_{\lambda}^{\tau}\left(n a_{t}\right)\left(e_{n-r+1} \wedge \ldots \wedge e_{n}\right) \mid\left(e_{n-r+1} \wedge \ldots \wedge e_{n}\right)\right\rangle= \\
& \left|\cosh t-e^{-t}\left(w+\frac{1}{2}[z, z]\right)\right|^{-2(n+1) \lambda}\left[\cosh t-e^{-t}\left(w+\frac{1}{2}[z, z]\right)\right]^{-r} \\
\cdot & \left\{\operatorname{det}\left(\begin{array}{cc}
I & -{ }_{r} \bar{z} e^{-t} \\
r & \cosh t+e^{-t}\left(w+\frac{1}{2}[z, z]\right)
\end{array}\right)-\left[\cosh t-e^{-t}\left(w+\frac{1}{2}[z, z]\right)\right]\right. \\
\cdot & \left.\operatorname{det}\left[\left(\begin{array}{c}
\sinh ^{-r} e^{-t}-e^{-t}\left(w+\frac{1}{2}[z, z]\right)
\end{array}\right) \cdot\left({ }_{r} \bar{z}^{*}, \sinh t+e^{-t}\left(w+\frac{1}{2}[z, z]\right)\right)\right]\right\}
\end{aligned}
$$

where ${ }_{r}^{t} z=\left(z_{n-r+2}, \ldots, z_{n}\right)$. The value of the first determinant is , by induction, seen to be equal to

$$
\cosh t+e^{-t}\left(w+\frac{1}{2}[z, z]\right)+\left(\left|z_{n-r+2}\right|^{2}+\ldots+\left|z_{n}\right|^{2}\right) e^{-t} \quad(r>1)
$$

while the second determinant vanishes for $r>1$. Hence

$$
\begin{aligned}
& \left\langle\psi_{\lambda}^{\tau}\left(n a_{t}\right)\left(e_{n-r+1} \wedge \ldots \wedge e_{n}\right) \mid\left(e_{n-r+1} \wedge \ldots \wedge e_{n}\right)\right\rangle= \\
= & \left|\cosh t-e^{-t}\left(w+\frac{1}{2}[z, z]\right)\right|^{-2(n+1) \lambda}\left[\cosh t-e^{-t}\left(w+\frac{1}{2}[z, z]\right)\right]^{-r} \\
& \cdot\left\{\cosh t+e^{-t}\left(w+\frac{1}{2}[z, z]\right)+\left(\left|z_{n-r+2}\right|^{2}+\ldots+\left|z_{n}\right|^{2}\right) e^{-t}\right\}
\end{aligned}
$$

In case $\tau_{n}$, we can just take $r=n$. And finally for $\sigma_{r}(1<r<n)$ we have:

$$
\begin{aligned}
& \left\langle\psi_{\lambda}^{\tau}\left(n a_{t}\right)\left(e_{1} \wedge \ldots \wedge e_{r}\right) \mid\left(e_{1} \wedge \ldots \wedge e_{r}\right)\right\rangle= \\
& \left|\cosh t-e^{-t}\left(w+\frac{1}{2}[z, z]\right)\right|^{-2(n+1) \lambda}\left[\cosh t-e^{-t}\left(w+\frac{1}{2}[z, z]\right)\right]^{-r} .
\end{aligned}
$$

Now we have to integrate these expressions (65)-(70) times $e^{-(\mu+2 \rho) t}$ over $n=n(z, w)$ and $t(-\infty<t<\infty)$, where $\mu \in \mathbb{C}$ is such that $\Phi_{\mu, \sigma}^{\tau}$ is positive definite. Here $z \in \mathbb{C}^{n-2}$, $w \in i \mathbb{R}$. By making some successive changes of variables we reduce the initial expressions to a combination of the following integrals:

$$
\begin{aligned}
F(\alpha, \beta, \gamma, \delta)= & \left.\int_{0}^{\infty} \int_{-\infty}^{\infty} \int_{\mathbb{C}^{n-1}} v^{\alpha}\left(1+v^{2}\left(1+|z|^{2}\right)\right)^{2}+4 v^{4}|w|^{2}\right)^{-\beta}|w|^{2 \gamma}|z|^{2 \delta} d v d w d z \\
= & 2^{-2 \gamma-3} S_{2 n-2} \Gamma(n+\delta-1) B\left(\frac{2 \gamma+1}{2}, 2 \beta-\frac{2 \gamma+1}{2}\right) \\
& \cdot \frac{\Gamma\left(\frac{\alpha}{2}+\frac{1}{2}-2 \gamma-n-\delta\right) \Gamma\left(2 \beta-\frac{\alpha}{2}-\frac{1}{2}\right)}{\Gamma(2 \beta-2 \gamma-1)}
\end{aligned}
$$


where $S_{n}=\frac{(2 \pi)^{[n / 2]}}{(n-2) ! !}$, we use here Lemma 8.10 .15 from [15]. Finally, we have that the spherical Fourier transform of (67) is equal to

$$
p_{1}(n, \lambda) \cdot \frac{\Gamma((n+1) \lambda+i \nu / 2-\rho / 2) \Gamma((n+1) \lambda-i \nu / 2-\rho / 2)}{\Gamma(2(n+1) \lambda+1)},
$$

where

$$
p_{1}(n, \lambda)=2^{2(n+1) \lambda-3} S_{2 n-2} \pi^{\frac{1}{2}} \frac{\Gamma(n-1) \Gamma\left(2(n+1) \lambda+\frac{1}{2}\right)}{\Gamma(2(n+1) \lambda+2)}\left(2(n+1) \lambda-\frac{3}{2}\right) .
$$

For (65) we have:

$$
p_{2}(n, \lambda) \cdot \frac{\Gamma((n+1) \lambda+i \nu / 2-\rho / 2) \Gamma((n+1) \lambda-i \nu / 2-\rho / 2)}{\Gamma(2(n+1) \lambda+3)},
$$

where

$$
\begin{aligned}
p_{2}(n, \lambda)= & 2^{2(n+1) \lambda+n} \frac{(2 n-3)}{(2 n) ! !} \pi^{n-\frac{1}{2}} \frac{\Gamma(n-1) \Gamma\left(2(n+1) \lambda+\frac{3}{2}\right)}{\Gamma(2(n+1) \lambda+4)} \\
& \cdot\left\{-|\nu|^{2}((n+1) \lambda+1)(n+1) \lambda+Q(n, \lambda)\right\}
\end{aligned}
$$

with $Q(n, \lambda)$ some polynomial in $\lambda$ and $n$.

The generic case $\left(\tau=\tau_{r}, r>1\right)$ is more complicated. Namely, we have the following expression for the Fourier transform of (70):

$$
\begin{aligned}
& c \frac{\Gamma((n 1) \lambda+i \nu / 2-\rho / 2) \Gamma((n+1) \lambda-i \nu / 2-\rho / 2)}{\Gamma(2(n+1) \lambda-1)} \cdot \sum_{k=0}^{r} \sum_{i=0}^{k} \sum_{j=0}^{[i / 2]} C_{r}^{k} C_{k}^{i} C_{i}^{2 j} \\
& \cdot(-1)^{j} \prod_{\alpha=1}^{i-2 j}(n-1+\alpha) \prod_{\beta=1}^{j}\left(j-\beta+\frac{1}{2}\right) \prod_{\gamma=0}^{r-j-1}\left(2(n+1) \lambda+\frac{3 r-1}{2}+\gamma\right) \\
& \frac{\prod_{r=0}^{r+k-i-1}((n+1) \lambda-\rho / 2+i \nu / 2+r) \prod_{s=0}^{r-k-1}((n+1) \lambda-\rho / 2-i \nu / 2+s)}{\prod_{t=0}^{r-2 j}(2(n+1) \lambda+t-1)}
\end{aligned}
$$

with

$$
c=\frac{2^{2(n+1) \lambda+r-3} S_{2 n-2} \pi^{\frac{1}{2}} \Gamma\left(2(n+1) \lambda+\frac{3 r}{2}-\frac{1}{2}\right)}{\Gamma(2(n+1) \lambda+2 r)} \Gamma(n-1) .
$$

But this expression is not satisfactory. In this case we shall use the Cartan decomposition $G=K A_{+} K$, the corresponding integral formula (see section 4.1) and the explicit expressions for the scalar components of $\psi_{\lambda}^{\tau}\left(a_{t}\right)$ and the $\tau$-spherical functions $\Phi_{\lambda}^{\tau}\left(a_{t}\right)$.

Let us recall that by Schur's Lemma any $\tau_{j}$-radial function $F$ is given by its scalar components $f_{\sigma}$ such that

$$
F\left(a_{t}\right)=\sum_{\sigma \in \hat{M}\left(\tau_{j}\right)}^{\oplus} f_{\sigma} I d_{V_{\sigma}} .
$$

Finally we have that the scalar components of $\psi_{\lambda}^{\tau}\left(a_{t}\right)$ are

$$
\begin{aligned}
\psi_{r-1}(t) & =(\cosh t)^{-2(n+1) \lambda-1}, \\
\psi_{r}(t) & =(\cosh t)^{-2(n+1) \lambda-2} .
\end{aligned}
$$


The scalar components of the $\tau$-spherical functions $\Phi_{\lambda}^{\tau}\left(a_{t}\right)$ are given by

$$
\begin{aligned}
\phi_{r-1}(\nu, t) & =(\cosh t)^{r+1}\left\{\frac{n}{r} H_{\nu}^{(n-1,1+r)}(t)-\frac{n-r}{r} H_{\nu}^{(n, r)}(t)\right\}, \\
\phi_{r}(\nu, t) & =(\cosh t)^{r} H_{\nu}^{(n, r)}(t),
\end{aligned}
$$

where $H_{\nu}^{(\alpha, \beta)}(t)={ }_{2} F_{1}\left(\frac{\alpha+\beta+1+i \nu}{2}, \frac{\alpha+\beta+1-i \nu}{2}, \alpha+1,-\sinh ^{2} t\right)$, see [2], Theorem 4.13. In fact, we slightly modified these formulæ because our representations $\tau_{r}$ differ from standard spinor representations by a some one-dimensional multiplicative factor, (see the section 7 of [2] for more details).

Applying the following formula (when it is well defined) (it can be obtained applying the results of [6], section 20.9)

$$
\begin{aligned}
& \int_{0}^{\infty}(1+x)^{-\lambda} x^{n-1}{ }_{2} F_{1}(a, b, n+1,-x) d x= \\
& \frac{\Gamma(n) \Gamma(\lambda+b-n)}{\Gamma(\lambda+b)}{ }_{3} F_{2}(n+1-a, n, b ; \lambda+b, n+1 ; 1) .
\end{aligned}
$$

we obtain the following expressions for the spherical Fourier transform of (69) and (70):

$$
\begin{aligned}
& -\hat{\phi}_{r-1}(\nu)=\frac{n}{2 r} \pi^{n} \frac{\Gamma\left((n+1) \lambda-\frac{\rho}{2}+\frac{1}{2}+\frac{i \nu}{2}\right) \Gamma\left((n+1) \lambda-\frac{\rho}{2}+\frac{1}{2}-\frac{i \nu}{2}\right)}{\Gamma\left((n+1) \lambda-\frac{r}{2}\right) \Gamma\left((n+1) \lambda+\frac{r}{2}+1\right)} \\
& -\pi^{n} \frac{n-r}{2 r} \frac{\Gamma\left((n+1) \lambda-\frac{\rho}{2}+\frac{1}{2}-\frac{i \nu}{2}\right)}{\Gamma\left((n+1) \lambda+\frac{\rho}{2}+\frac{1}{2}-\frac{i \nu}{2}\right)} \\
& { }_{3} F_{2}\left(\frac{\rho}{2}+\frac{1}{2}-\frac{r}{2}-\frac{i \nu}{2}, n, \frac{\rho}{2}+\frac{1}{2}+\frac{r}{2}-\frac{i \nu}{2},(n+1) \lambda+\frac{\rho}{2}+\frac{1}{2}-\frac{i \nu}{2}, \rho+1,1\right), \\
& \text { - } \hat{\phi}_{r}(\nu)=\frac{1}{2} \pi^{n} \frac{\Gamma\left((n+1) \lambda-\frac{\rho}{2}+\frac{3}{2}-\frac{i \nu}{2}\right)}{\Gamma\left((n+1) \lambda+\frac{\rho}{2}+\frac{3}{2}-\frac{i \nu}{2}\right)} \\
& { }_{3} F_{2}\left(\frac{\rho}{2}+\frac{1}{2}-\frac{r}{2}-\frac{i \nu}{2}, \rho, \frac{\rho}{2}+\frac{1}{2}+\frac{r}{2}-\frac{i \nu}{2},(n+1) \lambda+\frac{\rho}{2}+\frac{3}{2}-\frac{i \nu}{2}, \rho+1,1\right) .
\end{aligned}
$$

4.5. Decomposition of Berezin kernels of restrictions. We use the results obtained in the previous section (61)-(70). Notice that $n(w, z) \in H$ if and only if $w=0$ and $z \in \mathbb{R}^{n-2}$. We assume that $r \neq \frac{n}{2}$, then $\left.\tau_{r}\right|_{S O(n)}$ is irreducible and no discrete series enters in the Plancherel formula (see (4.2) and [11]). Finally, we have:

$$
\begin{aligned}
& -\left\langle\widehat{\Psi}_{\lambda}^{1} e_{1} \mid e_{1}\right\rangle(\nu) \\
& =2^{(n+1) \lambda-2} \frac{\Gamma\left((n+1) \lambda / 2+i \nu / 2+\rho^{\prime} / 2+\frac{1}{2}\right) \Gamma\left((n+1) \lambda / 2-i \nu / 2-\rho^{\prime} / 2+\frac{1}{2}\right)}{\Gamma((n+1) \lambda+2)} \\
& \cdot \Gamma((n-1) / 2) S_{n-2}(2((n+1) \lambda+1)+(n-1)(n-3) / 2), \\
& \text { - }\left\langle\widehat{\Psi}_{\lambda}^{1} e_{n} \mid e_{n}\right\rangle(\nu) \\
& =2^{(n+1) \lambda-2} S_{n-2} \frac{\Gamma\left((n+1) \lambda / 2+i \nu / 2-\rho^{\prime} / 2\right) \Gamma\left((n+1) \lambda / 2-i \nu / 2-\rho^{\prime} / 2\right)}{\Gamma((n+1) \lambda+1)} \\
& \cdot \Gamma((n-1) / 2)\left(((n+1) \lambda-n+1)+\frac{(n+1) \lambda+\frac{1}{2}+\frac{n^{2}-1}{4}-n-|\nu|^{2}}{(n+1) \lambda+1)}\right),
\end{aligned}
$$


- $\left\langle\widehat{\Psi}_{\lambda}^{r} e_{1} \wedge \ldots \wedge e_{r} \mid e_{1} \wedge \ldots \wedge e_{r}\right\rangle(\nu)$

$=2^{(n+1) \lambda+r-2} S_{n-2} \frac{\Gamma\left((n+1) \frac{\lambda}{2}+\frac{i \nu}{2}+\frac{r}{2}-\frac{\rho^{\prime}}{2}\right) \Gamma\left((n+1) \frac{\lambda}{2}-\frac{i \nu}{2}+\frac{r}{2}-\frac{\rho^{\prime}}{2}\right)}{\Gamma((n+1) \lambda+r)}$

$\cdot \Gamma((n-1) / 2)$,

- $\left\langle\widehat{\Psi}_{\lambda}^{r} e_{n-1+r} \wedge \ldots \wedge e_{n} \mid e_{n-1+r} \wedge \ldots \wedge e_{n}\right\rangle(\nu)$

$=2^{(n+1) \lambda+r-3} S_{n-2} \frac{\Gamma\left((n+1) \frac{\lambda}{2}+\frac{i \nu}{2}+\frac{r}{2}-\frac{\rho^{\prime}}{2}-\frac{1}{2}\right) \Gamma\left((n+1) \frac{\lambda}{2}-\frac{i \nu}{2}+\frac{r}{2}-\frac{\rho^{\prime}}{2}-\frac{1}{2}\right)}{\Gamma((n+1) \lambda+r)}$

$\left.\cdot \Gamma((n-1) / 2)\left((n+1) \lambda+r-\frac{n}{2}-\frac{1}{2}\right)+2(n-1)(r-2)\right)$.

Here $\rho^{\prime}$ is the half sum of the positive roots of $S O_{o}(1, n)$, so $\rho^{\prime}=\frac{n-1}{2}$.

\section{References}

[1] F. A. Berezin, Connection between co- and contravariant symbols of operators on the classical complex symmetric spaces, Dokl. Akad. Nauk SSSR 241 (1978), 15-17 (in Russian); English transl.: Soviet Math. Dokl. 19 (1978), 786-789.

[2] R. Camporesi, Harmonic analysis for spinor fields in complex hyperbolic spaces, Adv. Math. 154 (2000), 367-442.

[3] G. van Dijk and S. C. Hille, Maximal degenerate representations, Berezin kernels and canonical representations, in: Lie Groups and Lie Algebras, Their Representations, Generalizations and Applications. Kluwer Academic, Dordrecht, 1997.

[4] G. van Dijk and M. Pevzner, Berezin kernels on tube domains, J. Funct. Anal. 181 (2001), 189-209.

[5] G. van Dijk and M. Pevzner, Berezin kernels and maximal degenerate representations associated with Riemannian symmetric spaces of Hermitian type, in: Proc. NATO Adv. Study Inst., 2001, in press.

[6] A. Erdélyi et al., Tables of Integral Transforms, Vol 2, McGraw-Hill, New York, 1954.

[7] J. Faraut and G. Olafsson, Causal semisimple symmetric spaces, the geometry and harmonic analysis, in: Hofmann, Lawson and Vinberg (eds.), Semigroups in Algebra, Geometry and Analysis, de Gruyter, Berlin, 1995.

[8] S. Helgason, Differential Geometry, Lie Groups and Symmetric Spaces, Academic Press, New York, 1978.

[9] S. C. Hille, Canonical representations associated to a character on a Hermitian symmetric space of rank one, preprint Leiden University, 1996. See also: Thesis, Leiden University, 1999.

[10] T. H. Koornwinder, A note on the multiplicity free reduction of certain orthogonal and unitary groups, Indag. Math. 44 (1982), 215-218.

[11] E. Pedon, Analyse harmonique des formes différentielles sur l'espace hyperbolique réel, thesis, Université Henri Poincaré, Nancy, 1997.

[12] E. Pedon, Harmonic analysis for differential forms on complex hyperbolic spaces, J. Geom. Physics 32 (1999), 102-130.

[13] I. Satake, Algebraic Structures of Symmetric Domains, Iwanami Shoten and Princeton Univ. Press, 1980. 
[14] A. Unterberger and H. Upmeier, The Berezin transform and invariant differential operators, Comm. Math. Phys. 164 (1994), 563-597.

[15] N. R. Wallach, Harmonic Analysis on Homogeneous Spaces, Marcel Dekker, New York, 1973.

[16] G. Warner, Harmonic Analysis on Semi-simple Lie Groups II, Springer-Verlag, Berlin, 1972.

[17] G. Zhang, Berezin transform on line bundles over bounded symmetric domains, J. Lie Theory 10 (2000), 111-126. 